Research Article

\title{
Dynamic Characteristics and Restraint Mechanism of Elastically Restrained Connecting Joints on the Flexible Robotic Manipulator
}

\author{
Yufei Liu $\mathbb{D}^{1,2}$ Han Xu $\mathbb{D}^{3},{ }^{3}$ and Xi Zhang $\mathbb{D}^{1}$ \\ ${ }^{1}$ School of Mechanical and Automotive Engineering, Anhui Polytechnic University, Wuhu 241000, China \\ ${ }^{2}$ School of Mechatronic Engineering, China University of Mining and Technology, Xuzhou 221116, China \\ ${ }^{3}$ Tongji Zhejiang College, Jiaxing 314000, China \\ Correspondence should be addressed to Xi Zhang; zhangxicumt@163.com
}

Received 24 March 2018; Revised 5 August 2018; Accepted 3 September 2018; Published 26 September 2018

Academic Editor: Toshiaki Natsuki

Copyright (c) 2018 Yufei Liu et al. This is an open access article distributed under the Creative Commons Attribution License, which permits unrestricted use, distribution, and reproduction in any medium, provided the original work is properly cited.

Connecting joints are significant components in mechanical systems as well as the Cartesian flexible robotic manipulator (FRM) which consists of a flexible arm and a moving base through connecting joints. Uncertain factors, such as unqualified assembly, accidental collision, and longtime service, will reduce the restraint stiffness of the connecting joints and enhance the dynamic nonlinearity of the system subsequently. In this case, the traditional perfectly fixed restrained model cannot reflect the real property of the connecting joints. This paper focuses on the elasticity property of the connecting joints with uncertain restraint stiffness, which is defined as the elastically restrained connecting joints (ERCJ), and investigates the dynamic characteristics and restraint mechanism of the ERCJ. An elastic restrained model is proposed to describe the elasticity property of the connecting joints and determine the elastic restrained region of the ERCJ, and the frequency relationship equation in the elastic restrained region is simultaneously determined and verified. Based on the proposed elastic restrained model and Hamilton's variational principle, the dynamic model and vibration displacement equation of the FRM with elastically restrained connecting joints (FRMERCJ) are established. The virtual prototype experiment of the FRMERCJ is conducted to verify the dynamic model and reveal the restraint mechanism of the ERCJ. The proposed elastic restrained model in this paper can accurately describe the elasticity property of the connecting joints, and the ERCJ is sensitive to motion velocities especially under higher velocities for higher-order vibrations in the initial stage. The results are meaningful for the dynamic analysis and vibration control of robotic manipulators.

\section{Introduction}

Flexible manipulators have gradually applied in robotic systems [1-3]. Compared with rigid robotic manipulators (RRMs) constructed with heavy materials and bulky structures, flexible robotic manipulators (FRMs) are more lightweight and flexible and have been widely applied in aeronautics, astronautics, and precision manufacturing [4-6]. As shown in Figure 1, a Cartesian FRM can be modeled as a moving base, a flexible arm, and an end effector, which remains a typical rigid-flexible coupling system conveying rigid motions of the moving base and elastic vibrations of the flexible arm. This rigid-flexible coupling effect is transmitted through the connecting joints between the moving base and the flexible arm, for example, the bolted joints which are frequently used in mechanical systems and the Cartesian robotic systems because of their simple configuration, convenient operation, and low cost $[7,8]$. Due to their flexibility properties, FRMs will be more sensitive to this rigid-flexible coupling effect [9]. Therefore, the dynamic characteristic and effect of the connecting joints should be considered in the investigations of FRMs, especially for the accurate dynamic analysis and vibration control.

There have been numerous studies focused on the dynamic and vibration control of the FRMs [9]. Aiming at the Cartesian robotic system, Dadfarnia et al. [10] and Qiu [11] 


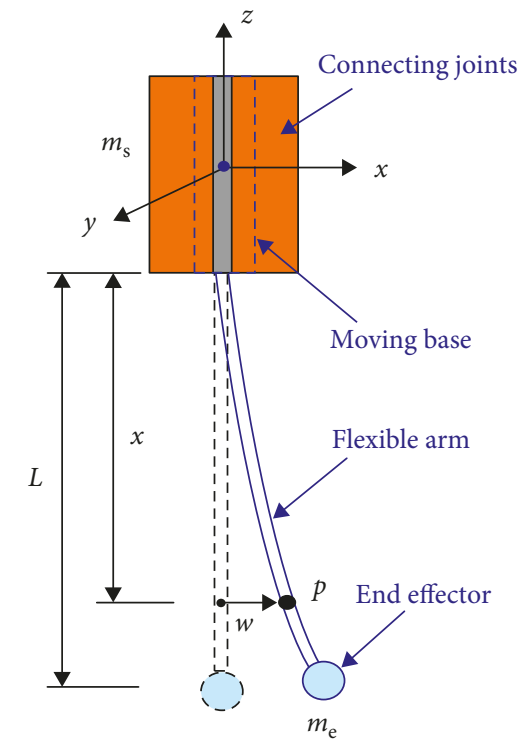

FIGURE 1: Structure diagram of the FRM.

studied the vibration control of the FRM with piezoelectric materials. Liu et al. [12] investigated the dynamic of a motordriven FRM system under the effect of electromechanical coupling factors in the driving system. Lu et al. [13] proposed a composite control of a rotational FRM with trajectory tracking of the driving system and vibration suppression of the flexible arm. The flexible arms in these indicated investigations are commonly installed on the moving base through bolted joints, and the bolted joints in the dynamic model are regarded as absolutely fixed restrained joints without considering the elastic or flexible property of the connecting joints, which is difficult to achieve in actual applications. Actually, the connecting stiffness of the bolted joints is determined by the preloads in the assembling process; for the situations that the preloads are not accurately controlled or undergoing accidental collision and longtime executions, the connecting stiffness of the bolted joints will simultaneously reduce smaller than the expected values. Chen et al. [14] indicate that the simplified tightening process may cause a smaller resultant torque due to the opposite direction of the two torque components, which will lead to a greater loss of preload than the value in reality, and it reveals that there exists a creep slip phenomenon on the bolt head bearing surface, which causes the bolt self-loosening to occur. This insufficient connecting stiffness deviates the ideal status and exhibits certain elasticity, which enhances the rigid-flexible coupling effect between the flexible arm and the moving base accordingly [15]. The rigid-flexible coupling effect aroused by these elastically restrained connecting joints (ERCJ) will be more sensitive to the disturbance excitations during operations and influence the dynamic characteristics of the FRM [16].

The joint flexibilities or elasticity in the robotic system has attracted increasingly wide attentions from researchers. Subudhi and Morris [17] discussed the dynamic modeling, simulation, and control of a manipulator with flexible links and joints. Qian and Zhang [18] and Malgaca et al. [19] investigated the effect of joint flexibility on the dynamic characteristics of the manipulator. Wei et al. [20] proposed a global mode method to obtain the reduced-order analytical dynamic model for a signal flexible-link flexible-joint manipulator. Using link and joint position measurement, Agee [21] proposed a distributed higher-order differential feedback controller to reduce the joint vibration and improve the tracking behavior in reference trajectory tracking control. The joint flexibilities investigated in these existing literatures are commonly focused on the joints between the links and are different with the connecting joints between the flexible arm and the moving base which will be discussed in this paper. The existing researches on these bolted connecting joints mainly focus on such large structures as machine tools and bridge structures, and however, there are very little about robotic systems. On the contrary, the bolted connecting joints in these large structures are generally static and the investigations are mainly about stiffness identification and structural optimization [22, 23]. However, to comprehensively assess the elasticity property of the connecting joints, the dynamic characteristics and restraint mechanism of the connecting joints for the structure undergoing movements of the robotic system should be revealed. In our previous studies, the vibration characteristics of the flexible arm with elastically restrained connecting joints were studied $[23,24]$, and however, the connecting joints studied are stationary without the influence of movements. Moreover, to accurately reflect the elasticity property of the connecting joints, the elastic restrained region as well as the internal relationship equation of the restraint stiffness and the modal characteristics should be revealed. For this, the dynamic restraint mechanism of the elastically restrained connecting joints remains a further study.

In this paper, aiming at the connecting joints between the flexible arm and the moving base and considering their elasticity property, an accurate dynamic model will be established to investigate the coupling dynamic characteristics of the FRM system. The elastic restrained region and the definite frequency relationship equation in this elastic restrained region will be determined. Furthermore, the dynamic restraint mechanism of the elastically restrained connecting joints under movements is investigated. The remainder of this paper is organized as follows. In Section 2, the mode frequency characteristic of the FRM with elastically restrained connecting joints (FRMERCJ) is investigated and verified experimentally, which conducts the frequency relationship equation in the elastic restrained region. Considering the elasticity property of the connecting joints, the vibration response characteristics of the FRMERCJ are investigated in Section 3. Base on virtual prototype experiment, the dynamic restraint mechanism of the elastically restrained connecting joints is revealed in Section 4. The paper is concluded with a brief summary in Section 5 .

\section{Mode Frequency Characteristic of the FRMERCJ}

2.1. Mode Frequency Characteristic. As the foundation of dynamic analysis, the mode frequency characteristic will be investigated in this section. According to the structure 
diagram of the Cartesian FRM in Figure 1, to describe the elasticity property of the connecting joints caused by insufficient connecting stiffness, the restraint of the connecting joints is modeled as tensional springs with restraint rigidities $k$, and the elastic restrained model can be constructed as shown in Figure 2. In this case, the FRM remains a rigidflexible coupling system.

Based on the elastic restrained model in Figure 2, the orthogonal mode shape of the FRMERCJ can be denoted as [25]

$$
\varphi(x)=\alpha_{1} \sin \beta x+\alpha_{2} \cos \beta x+\alpha_{3} \sinh \beta x+\alpha_{4} \cosh \beta x,
$$

where $\alpha_{1}, \alpha_{2}, \alpha_{3}$, and $\alpha_{4}$ are the coefficients determined by boundary conditions and $\beta$ is a related parameter of frequency $\omega$ and can be determined as

$$
\beta^{2}=\sqrt{\frac{\rho A}{E I}} \omega,
$$

where $\rho$ is the mass density, $A$ is the cross-sectional area, $A=b \times h, b$ and $h$ are the width and the thickness, respectively, $E$ is Young's modulus, and $I$ is the cross-sectional moment of inertia about the neural axis, $I=b h^{3} / 12$.

According to Reference [16], the mode frequency equation of the FRMERCJ can be described as

$$
\begin{gathered}
\frac{E I \beta^{3}}{k}(\cos \beta L \sinh \beta L+\sin \beta L \cosh \beta L) \\
-(\cos \beta L \cosh \beta L+1)=0 .
\end{gathered}
$$

In Equation (3), assuming the restraint stiffness $k \longrightarrow$ $+\infty$, the frequency equation will be converted to $\cos \beta L \cosh \beta L+1=0$ which is the frequency equation of the flexible robotic manipulator with fixed restrained connecting joints (FRMFRCJ).

Numerical simulation is conducted to investigate the mode frequency characteristic of the FRMERCJ with different restraint stiffnesses. In the simulation, the flexible arm is characterized as a flexible beam to describe its lower damp and stiffness features with following structural parameters: length $L=400 \mathrm{~mm}$, width $b=45 \mathrm{~mm}$, thickness $h=3 \mathrm{~mm}$, Young's modulus $E=25.24 \mathrm{GPa}$, volumetric density $\rho=2030 \mathrm{~kg} / \mathrm{m}^{3}$, Poisson's ratio $\mu=0.30$, and mass of the damping ratio $\zeta=0.005$.

Figure 3 presents the first three orders mode frequencies of the FRMERCJ. It can be seen that with increasing of the restraint stiffness, the mode frequencies gradually increase and close to the situation of FRMFRCJ, with the frequencies of $10.68 \mathrm{~Hz}, 66.93 \mathrm{~Hz}$, and $187.41 \mathrm{~Hz}$. It can be obtained that the ideal fixed restrained connecting joints are difficult to achieve, and the restraint stiffness of the connecting joints is actually smaller.

From Figure 3, the interval $\left[10^{4} \mathrm{~N} / \mathrm{m}, 10^{6} \mathrm{~N} / \mathrm{m}\right]$ can be regarded as the elastic restrained region of the connecting joints as indicated in the figure. It can be found that, in this elastic restrained region, the relationship between the mode frequency and the restraint stiffness conveys the characteristic of the power function. Therefore, the power function

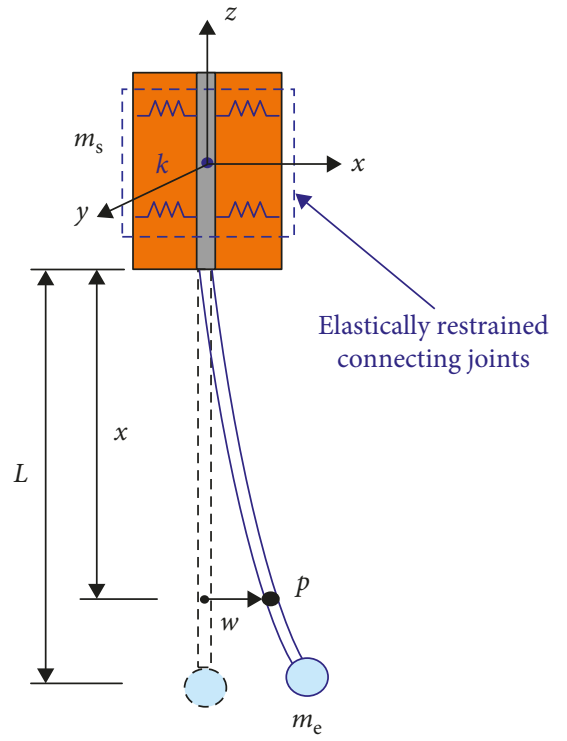

FIgURE 2: Elastic restrained model of the FRM.

is applied to fit the frequency fitting equation in the elastic restrained region, which can be expressed as

$$
\omega_{0 i}=a_{i} k^{b_{i}}+c_{i}, \quad i=1,2,3 .
$$

Using the fitting tool cftool in Matlab software, the coefficients of the fitting equation of the first three orders mode frequencies are obtained as $a_{1}=-2173, a_{2}=-72570$, $a_{3}=-11650, \quad b_{1}=-0.9973, \quad b_{2}=-0.9152, \quad b_{3}=-0.5334$, $c_{1}=10.68, c_{2}=67.07$, and $c_{3}=195.5$, respectively. Figure 4 presents the error analysis of the first three orders mode frequencies obtained by the frequency fitting equation. It can be seen that the fitting errors of the first- and second-order frequencies are smaller than $0.08 \%$ and $0.45 \%$, respectively, and the fitting error of the third-order frequency is greater than the first and second orders for lower restraint stiffness, with the increase of restraint stiffness, and the fitting errors will be significantly reduced.

2.2. Experimental Verification. To verify the elastic restrained model, a modal test experiment device is setup as shown in Figure 5. The connecting joints are modeled using bolted joints. The modal analysis model in the modal test experiment is established with ten divided segments in the length and two divided segments in the width. The measurement points are arranged that point 3 is the vibration pickup point and other points are the incentive points. The experiment is conducted using the approach of single-point vibration pickup with a force hammer. The piezoelectric acceleration sensor DH131 is installed on the position of point 3 to obtain vibration signals, and the mass of the piezoelectric acceleration sensor is $m_{\mathrm{e}}=1 \mathrm{~g}$. The charge modulator DH5857-1 is applied to achieve the vibration signal processing. The modal analysis software is the dynamic signal acquisition and analysis system DH5927N.

According to the modal test experiment, the first three orders frequencies of the FRM are $9.12 \mathrm{~Hz}, 55.59 \mathrm{~Hz}$, and 


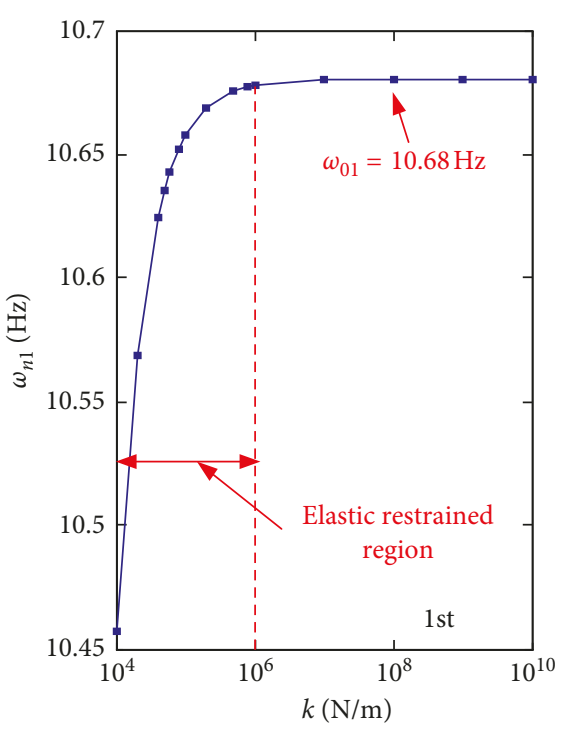

(a)

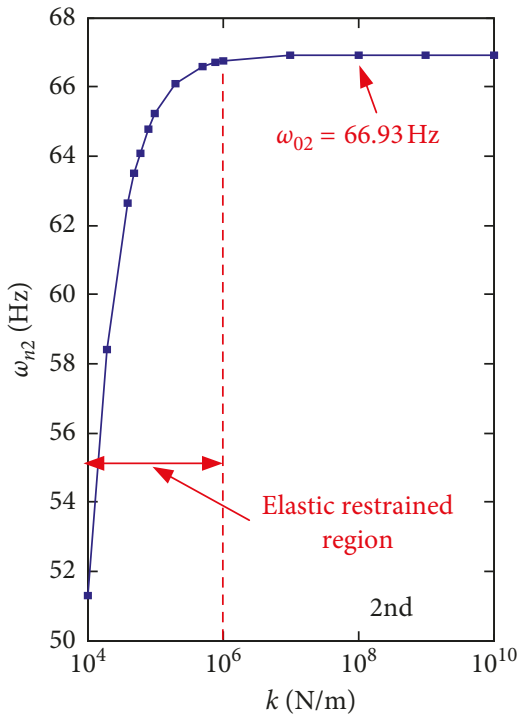

(b)

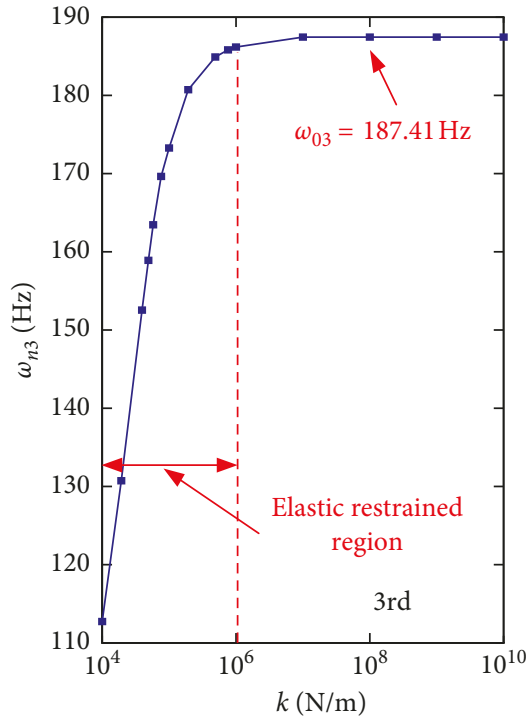

(c)

Figure 3: The first three orders mode frequencies of the FRMERCJ.

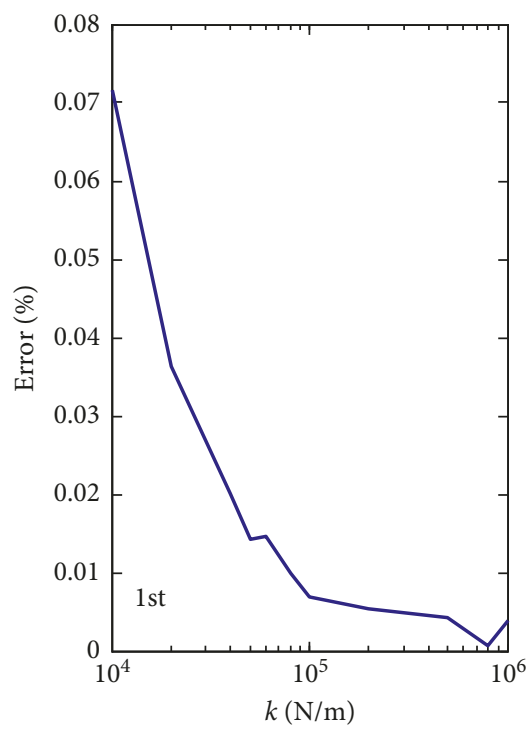

(a)

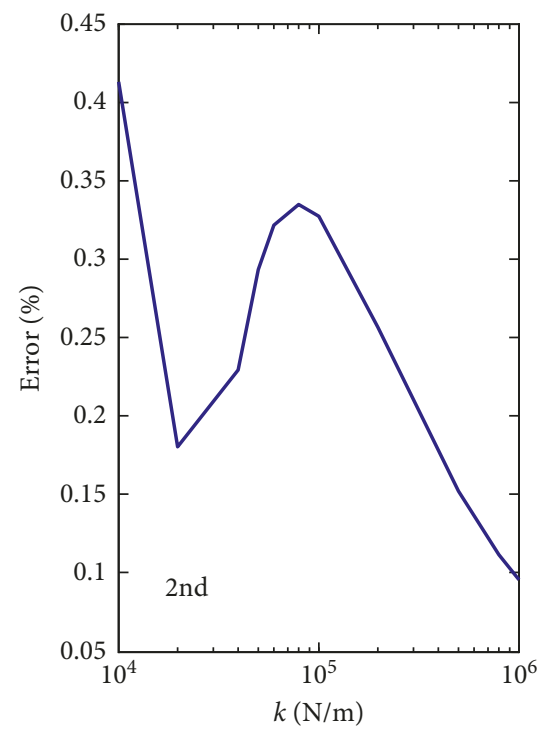

(b)

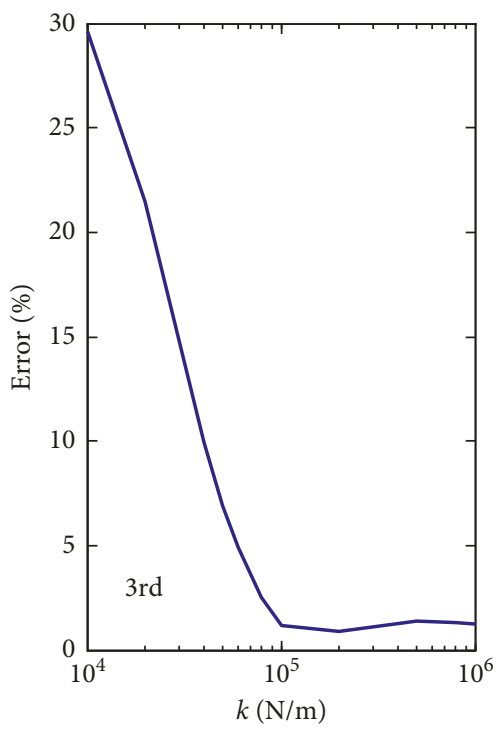

(c)

Figure 4: Error analysis of the frequencies fitting the equation.

$151.85 \mathrm{~Hz}$, respectively, which obviously deviate than that of the FRMFRCJ. The frequency error function is defined as

$$
\varepsilon_{\omega}=\sum_{i=1}^{3}\left(\omega_{0 i}-\omega_{i}\right)^{2} .
$$

Using the fminbnd optimization function in Matlab software, the restraint stiffness corresponding to the experimental modal results is obtained as $k=3.4 \times 10^{4} \mathrm{~N} / \mathrm{m}$, which denotes the restraint stiffness of the connecting joints in the FRMERCJ. According to Equation (3), the first three orders frequencies of the FRMERCJ are $10.61 \mathrm{~Hz}, 61.84 \mathrm{~Hz}$, and $150.60 \mathrm{~Hz}$.
To illustrate the analysis accuracy of the proposed elastic restrained model, the natural frequencies of the FRM under different restrained models and analysis errors of different mode orders are shown in Figure 6. It can be obtained that, with the increase in mode orders, the error of the elastic restrained model reduces while the fixed restraint model increases, and the results of the elastic restrained model are close to the experimental results with a relatively smaller error than the fixed restrained model, which indicates that the proposed elastic restrained model can achieve more accurate results especially for higher-order modes. To comprehensively illustrate the accuracy and reliability of the proposed elastic restrained model, the rms errors of the 


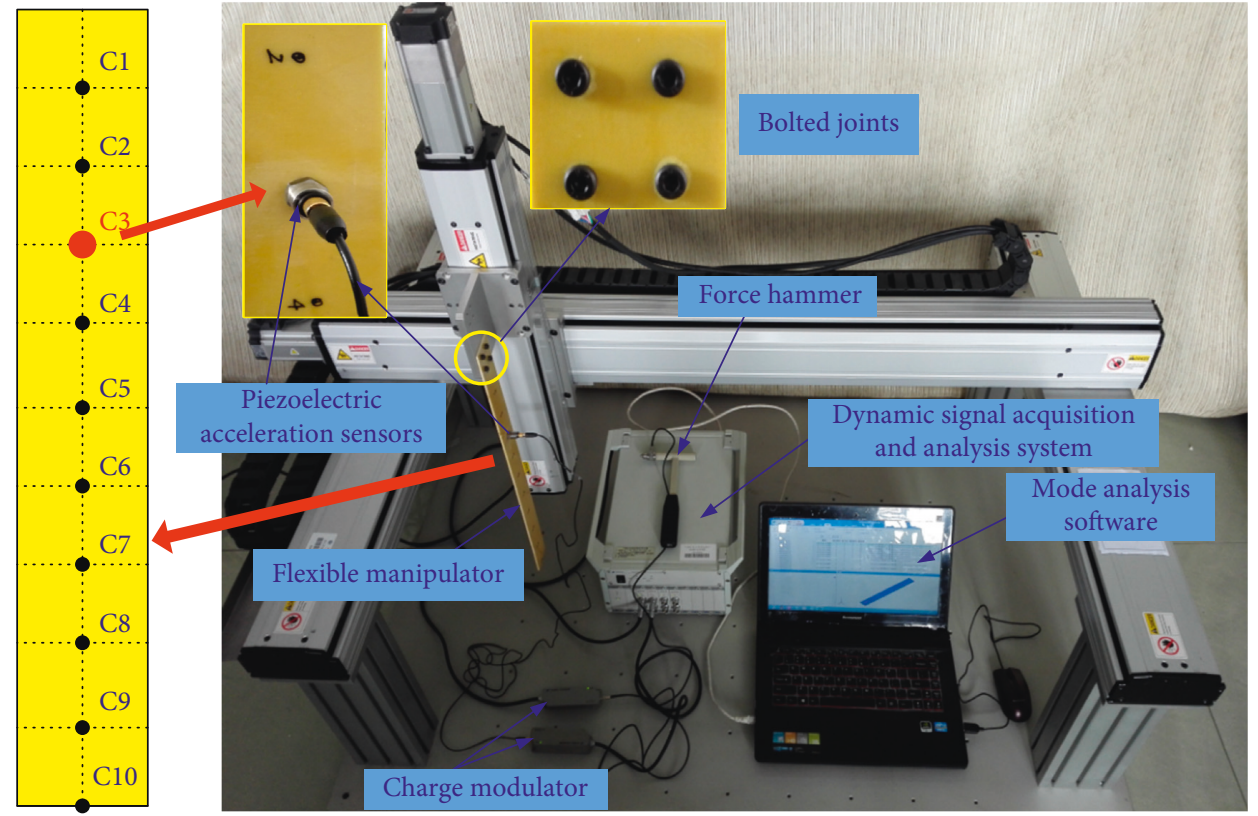

Figure 5: Modal experiment device and measurement point arrangement.

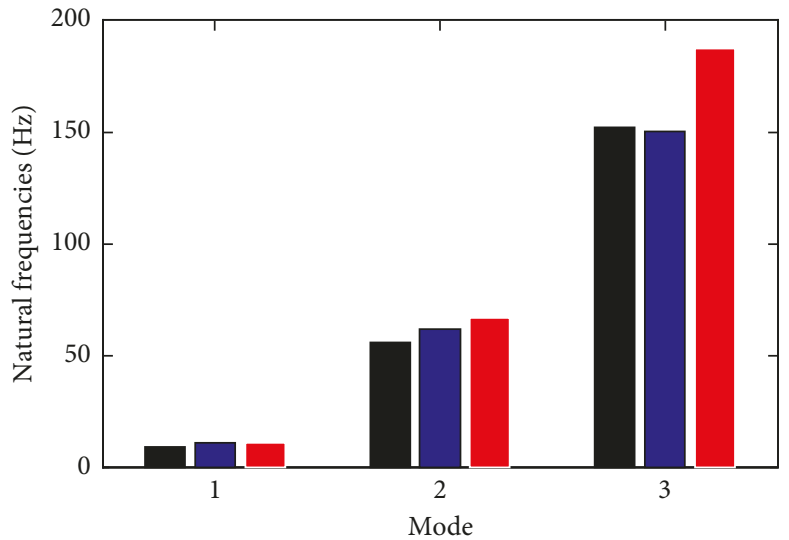

Experiment

Elastic restrained model

Fixed restrained model

(a)

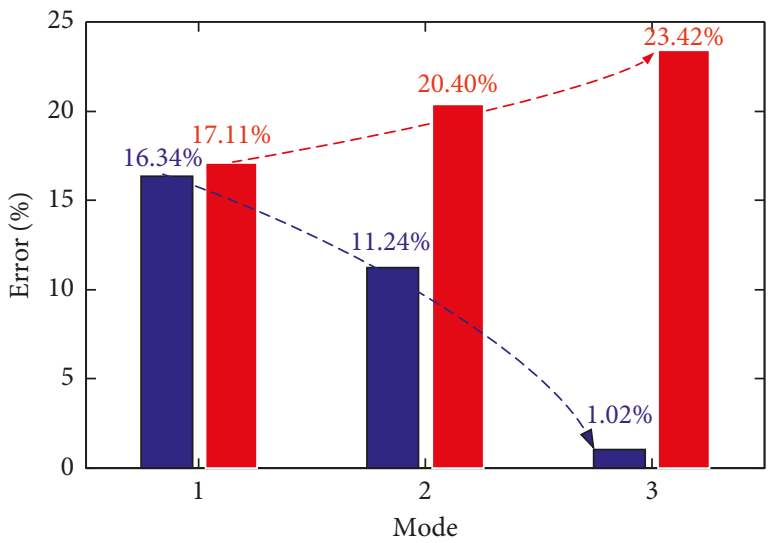

Elastic restrained model

Fixed restrained model

(b)

FIGURE 6: Natural frequencies and error analysis of the FRM with different restrained models: (a) natural frequencies; (b) analysis error.

natural frequency analysis results under different restrained models are calculated, and the values of the elastic restrained model and the fixed restrained model are 3.8160 and 21.5681, respectively, which further indicates that the proposed elastic restrained model has a better performance of accuracy and reliability.

\section{Vibration Response Characteristic of the FRMERCJ}

3.1. Vibration Equation Based on Hamilton's Variational Principle. As shown in Figure 2, the FRMERCJ remains a rigid-flexible coupling dynamic system, and the FRM conveys coupling motions, namely, rigid motions of the moving base and the elastic vibrations of the flexible arm. In this section, based on the proposed elastic restrained model, the rigid-flexible coupling dynamic model will be established to investigate the vibration characteristic of the FRMERCJ and restraint mechanism of the ERCJ. During the dynamic modeling, assumptions are made that the flexible arm satisfies the Bernoulli-Euler beam assumptions neglecting the shear deformations and the nonlinear coupling terms, and the transverse vibrations in the translating movements are the primary motions. 
The kinetic energy of the FRMERCJ can be written as

$$
E_{\mathrm{T}}=\frac{1}{2} m_{\mathrm{s}} v^{2}+\frac{1}{2} \rho A \int_{0}^{L}\left(v+\frac{\partial w}{\partial t}\right)^{2} d x+\frac{1}{2} m_{\mathrm{e}}\left(v+\left.\frac{\partial w}{\partial t}\right|_{x=L}\right)^{2},
$$

where $v$ denotes the velocity of translating movement, $m_{\mathrm{s}}$ is the mass of the moving base, $m_{\mathrm{e}}$ is the mass of the end effector, and $w(x, t)$ represents the transverse vibration displacements. The first part represents the kinetic energy of the moving base, the second part represents the kinetic energy of the flexible arm, and the third term indicates the kinetic energy of the end effector.

The potential energy of the FRMERCJ mainly considers the bending elastic potential energy of the flexible arm and can be written as

$$
E_{\mathrm{V}}=\frac{1}{2} E I \int_{0}^{L}\left(\frac{\partial^{2} w}{\partial x^{2}}\right)^{2} d x .
$$

Considering the damping effect of the flexible arm, the virtual work can be represented as

$$
\delta W=-v_{s} \int_{0}^{L} \frac{\partial w}{\partial t} \delta w d x,
$$

where $v_{\mathrm{s}}$ is the Rayleigh structural damping, $v_{s}=2 \xi_{i} \omega_{i}$, where $\zeta_{i}$ and $\omega_{i}$ are the $i$ th damping ratio and modal frequency, respectively.

To establish the dynamic model of the FRMERCJ, the Lagrangian function is defined as

$$
U=E_{\mathrm{T}}-E_{\mathrm{V}}+W .
$$

and according to Hamilton's principle [26], Equation (9) satisfies the following condition:

$$
\delta \int_{t_{0}}^{t_{1}} U d t=\int_{t_{0}}^{t_{1}} \delta\left(E_{\mathrm{T}}-E_{\mathrm{V}}+W\right) d t=0,
$$

where

$$
\begin{aligned}
\delta E_{\mathrm{T}}= & \delta\left\{\frac{1}{2} m_{\mathrm{s}} v^{2}+\frac{1}{2} \rho A \int_{0}^{L}\left(v+\frac{\partial w}{\partial t}\right)^{2} d x\right. \\
& \left.+\frac{1}{2} m_{\mathrm{e}}\left(v+\left.\frac{\partial w}{\partial t}\right|_{x=L}\right)^{2}\right\} \\
= & \rho A \int_{0}^{L}\left(v+\frac{\partial w}{\partial t}\right) \frac{\partial}{\partial t} \delta w d x+m_{\mathrm{e}}\left(v+\left.\frac{\partial w}{\partial t}\right|_{x=L}\right) \frac{\partial}{\partial t} \delta w \\
= & -\rho A \int_{0}^{L}\left(\dot{v}+\frac{\partial^{2} w}{\partial t^{2}}\right) \delta w d x-m_{\mathrm{e}}\left(\dot{v}+\left.\frac{\partial^{2} w}{\partial t^{2}}\right|_{x=L}\right) \delta w \\
\delta E_{\mathrm{V}}= & \delta\left\{\frac{1}{2} E I \int_{0}^{L}\left(\frac{\partial^{2} w}{\partial x^{2}}\right)^{2} d x\right\}=E I \int_{0}^{L} \frac{\partial^{2} w}{\partial x^{2}} \frac{\partial^{2}}{\partial x^{2}} \delta w d x \\
= & E I \int_{0}^{L} \frac{\partial^{4} w}{\partial x^{4}} \delta w d x .
\end{aligned}
$$

Substituting Equations (11) and (12) into Equation (10) yields

$$
\begin{aligned}
\delta \int_{t_{0}}^{t_{1}} U d t= & \int_{t_{0}}^{t_{1}}\left(\delta E_{\mathrm{T}}-\delta E_{\mathrm{V}}+\delta W\right) d t \\
= & -\int_{t_{0}}^{t_{1}}\left[\int_{0}^{L}\left(\rho A \dot{v}+\rho A \frac{\partial^{2} w}{\partial t^{2}}+E I \frac{\partial^{4} w}{\partial x^{4}}\right) d x\right. \\
& \left.+m_{\mathrm{e}}\left(\dot{v}+\left.\frac{\partial^{2} w}{\partial t^{2}}\right|_{x=L}\right)+v_{\mathrm{s}} \int_{0}^{L} \frac{\partial w}{\partial t} d x\right] \delta w d t \\
= & 0 .
\end{aligned}
$$

According to Equation (13), the following equation is obtained:

$$
\begin{aligned}
& \int_{0}^{L}\left[\rho A\left(\dot{v}+\frac{\partial^{2} w}{\partial t^{2}}\right)+v_{\mathrm{s}} \frac{\partial w}{\partial t}+E I \frac{\partial^{4} w}{\partial x^{4}}\right] d x \\
& \quad=-m_{\mathrm{e}}\left(\dot{v}+\left.\frac{\partial^{2} w}{\partial t^{2}}\right|_{x=L}\right) .
\end{aligned}
$$

To obtain the vibration displacements, according to the assumed mode method [25], the transverse vibration displacements $w(x, t)$ can be expressed as

$$
w(x, t)=\sum_{i=1}^{n \longrightarrow} \varphi_{i}(x) q_{i}(t)
$$

where $q_{i}(t)$ denotes the $i$ th generalized coordinate and $\phi_{i}(x)$ denotes the $i$ th orthogonal mode shape which can be descried as

$$
\begin{aligned}
\varphi(x)= & \sin \beta x-\sinh \beta x+\frac{2 E I \beta^{3}}{k} \cosh \beta x \\
& +\zeta_{*}(\cos \beta x-\cosh \beta x)
\end{aligned}
$$

where

$$
\zeta_{*}=-\frac{k(\sin \beta L+\sinh \beta L)+\lambda \beta(\cos \beta L-\cosh \beta L)}{k(\cos \beta L+\cosh \beta L)-\lambda \beta(\sin \beta L+\sinh \beta L)} .
$$

According to the orthogonality of mode shapes [27], it yields $\int_{0}^{L} \phi_{i}^{2} d x=1$, and Equations (8), (11), and (12) can be converted into

$$
\begin{aligned}
& \delta W=-\sum_{i} v_{\mathrm{si}} \dot{q}_{i} \delta q_{i} \\
& \delta E_{\mathrm{T}}=-\sum_{i}\left\{\left[m_{\mathrm{e}} \varphi_{i}^{2}(L)+\rho A\right] \ddot{q}_{i}+\left[m_{\mathrm{e}} \varphi_{i}(L)+m_{i}\right] \dot{v}\right\} \delta q_{i} \\
& \delta E_{\mathrm{V}}=\int_{0}^{L} E I \frac{\partial^{4} w}{\partial x^{4}} \delta w d x=\sum_{i} \int_{0}^{L} E I \frac{d^{4} \phi_{i}}{d x^{4}} q_{i} \phi_{i} \delta q_{i} d x
\end{aligned}
$$

where $m_{i}=\int_{0}^{L} \rho A \varnothing_{i} d x,(\bullet)$ denotes the time derivative.

Substituting Equations (18)-(20) into Equation (10) yields 


$$
\begin{aligned}
\delta \int_{t_{0}}^{t_{1}} U d t & =\int_{t_{0}}^{t_{1}}\left(\begin{array}{c}
-\sum_{i}\left\{\left[m_{\mathrm{e}} \varphi_{i}^{2}(L)+\rho A\right] \ddot{q}_{i}+\left[m_{\mathrm{e}} \varphi_{i}(L)+m_{i}\right] \dot{v}\right\} \delta q_{i} \\
-\sum_{i} \rho A \omega_{i}^{2} q_{i} \delta q_{i}-\sum_{i} v_{s i} \dot{q}_{i} \delta q_{i}
\end{array}\right) d t, \\
& =-\int_{t_{0}}^{t_{1}} \sum_{i}\left\{\left[m_{\mathrm{e}} \varphi_{i}^{2}(L)+\rho A\right] \ddot{q}_{i}+v_{\mathrm{si}} \dot{q}_{i}+\rho A \omega_{i}^{2} q_{i}+\left[m_{\mathrm{e}} \varphi_{i}(L)+m_{i}\right] \dot{v}\right\} \delta q_{i} d t=0 .
\end{aligned}
$$

According to Equation (21), the vibration displacement equation in generalized coordinate of the FRMERCJ can be obtained as

$$
\left[m_{\mathrm{e}} \varphi_{i}^{2}(L)+\rho A\right] \ddot{q}_{i}+v_{\mathrm{si}} \dot{q}_{i}+\rho A \omega_{i}^{2} q_{i}+\left[m_{\mathrm{e}} \varphi_{i}(L)+m_{i}\right] \dot{v}=0 .
$$

And via Duhamel integral [25], the following equation is obtained:

$$
\begin{aligned}
q_{i}(t)= & e^{-\zeta_{i} \omega_{* t i} t}\left(b_{1 i} \cos \sqrt{1-\zeta_{i}^{2}} \omega_{* t i} t+\frac{b_{2 i}+b_{1 i} \zeta_{i} \omega_{* t i}}{\sqrt{1-\zeta_{i}^{2}} \omega_{* t i}}\right. \\
& \left.\cdot \sin \sqrt{1-\zeta_{i}^{2}} \omega_{* t i} t\right)+\frac{f_{i}}{\sqrt{1-\zeta_{i}^{2}} \omega_{* t i}} \\
& \cdot \int_{0}^{t} \dot{v}(\tau) e^{-\zeta_{i} \omega_{* t i}(t-\tau)} \sin \omega_{* t i}(t-\tau) d \tau
\end{aligned}
$$

where

$$
\begin{aligned}
b_{1 i} & =\int_{0}^{L} w(x, 0) \varphi_{i} d x, \\
b_{2 i} & =\int_{0}^{L} \frac{\partial w(x, 0)}{\partial t} \varphi_{i} d x, \\
\omega_{* t i}^{2} & =\frac{\rho A \omega_{i}^{2}}{m_{\mathrm{e}} \varphi_{i}^{2}(L)+\rho A}, \\
f_{i} & =-\frac{m_{\mathrm{e}} \varphi_{i}(L)+m_{i}}{m_{\mathrm{e}} \varphi_{i}^{2}(L)+\rho A} .
\end{aligned}
$$

Based on Equations (15) and (23), the vibration displacement of the FRMERCJ can be obtained as

$$
\begin{aligned}
w(x, t)= & \sum_{i=1}^{n \longrightarrow \infty}\left[e ^ { - \zeta _ { i } \omega _ { * i } t } \left(b_{1 i} \cos \sqrt{1-\zeta_{i}^{2}} \omega_{* i} t+\frac{b_{2 i}+b_{1 i} \zeta_{i} \omega_{* i}}{\sqrt{1-\zeta_{i}^{2}} \omega_{* i}}\right.\right. \\
& \left.\cdot \sin \sqrt{1-\zeta_{i}^{2}} \omega_{* i} t\right)+\frac{f_{i}}{\sqrt{1-\zeta_{i}^{2}} \omega_{* t i}} \\
& \left.\cdot \int_{0}^{t} \dot{v}(\tau) e^{-\zeta_{i} \omega_{* t i}(t-\tau)} \sin \omega_{* t i}(t-\tau) d \tau\right] \varphi_{i}(x) .
\end{aligned}
$$

3.2. Vibration Response Characteristics of the FRMERCJ. In this section, the vibration response characteristics of the FRMERCJ are presented to reveal the influence of the ERCJ. The structural parameters of the flexible arm in this section are consistent with that in Section 2.1, and the vibrations at the position of the end effector are selected for the analysis.

Figure 7 presents the vibration responses of the FRMERCJ with different restraint stiffnesses. In the simulation, the velocity of the moving base is assigned as $v=0.01 \mathrm{~m} / \mathrm{s}$, and the restraint stiffness is assigned as $k=1.0 \times 10^{5} \mathrm{~N} / \mathrm{m}$, $1.0 \times 10^{6} \mathrm{~N} / \mathrm{m}$, and $1.0 \times 10^{7} \mathrm{~N} / \mathrm{m}$. It indicates that the vibration amplitudes enhanced with the decrease of restraint stiffness, and the vibration responses of the FRMERCJ are noticeable with larger vibration amplitudes than the FRMFRCJ, which is noticeable in the initial stage. It can be obtained that the elasticity property of the connecting joints has significant influence on the vibration responses with enhancing the vibrations especially in the initial stage.

To explain the vibration enhancing phenomenon in the initial stage indicated in Figure 7, which is related with the initial conditions, the trapezoid acceleration property of the driving system is considered in this section. The motion involves two processes, namely, start-up accelerating process and uniform running process. For the same start-up acceleration, the accelerating time $\Delta t$ is determined by the target velocity $v$. In this paper, the start-up acceleration is assigned as $1 \mathrm{~m} / \mathrm{s}^{2}$, and the FRM can obtain the target velocities of $0.01 \mathrm{~m} / \mathrm{s}, 0.02 \mathrm{~m} / \mathrm{s}$, and $0.03 \mathrm{~m} / \mathrm{s}$ with accelerating time $\Delta t=0.01 \mathrm{~s}, 0.02 \mathrm{~s}$, and $0.03 \mathrm{~s}$, respectively. Figures 8 and 9 present the vibration displacements and vibration velocities in the start-up accelerating process, which indicate that the ERCJ have noticeable influence on the initial vibration displacement and vibration velocity.

To comprehensively assess the elasticity property of the connecting joints, the dynamic characteristics of the connecting joints considering the movements of the robotic system should be revealed. Figure 10 further shows the vibration responses of the FRMERCJ with different motion velocities, which are assigned as $v=0.01 \mathrm{~m} / \mathrm{s}, 0.02 \mathrm{~m} / \mathrm{s}$, and $0.03 \mathrm{~m} / \mathrm{s}$, and the restraint stiffness is assigned as $k=1.0 \times 10^{6} \mathrm{~N} / \mathrm{m}$. It can be seen that, with the increasing of velocities, the vibration displacements of the FRMERCJ increase obviously while the FRMFRCJ present a minor change. This indicates that the ERCJ are sensitive to the motion velocities, and the influence of elasticity property will be enhanced under higher velocities. 


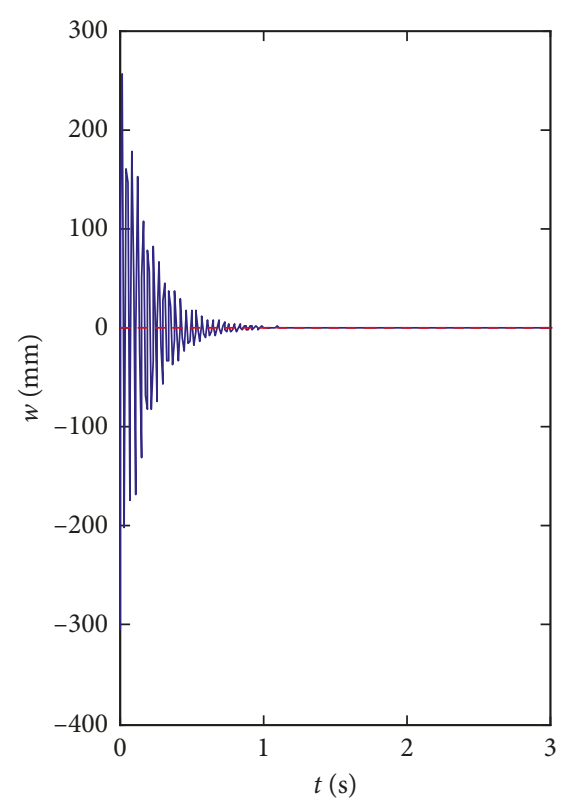

- $k=1.0$ times $10^{5} \mathrm{~N} / \mathrm{m}$

- - - Ideal fixed restraint

(a)

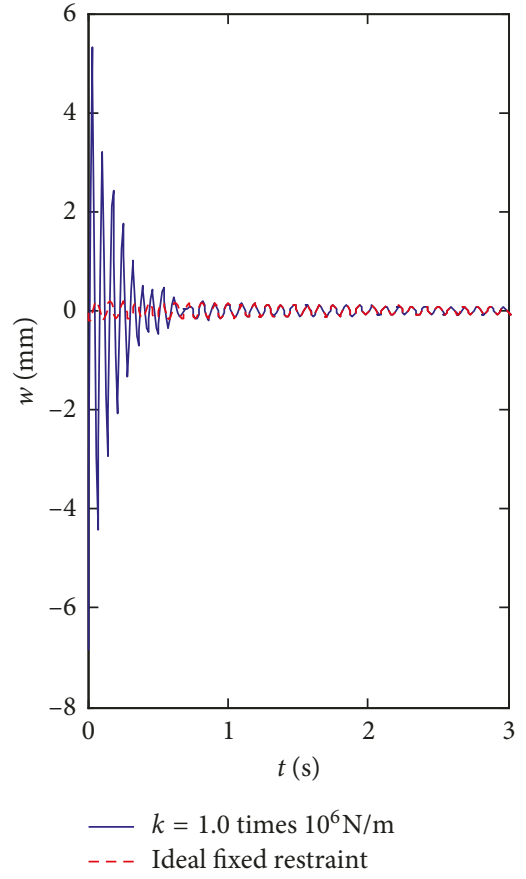

(b)

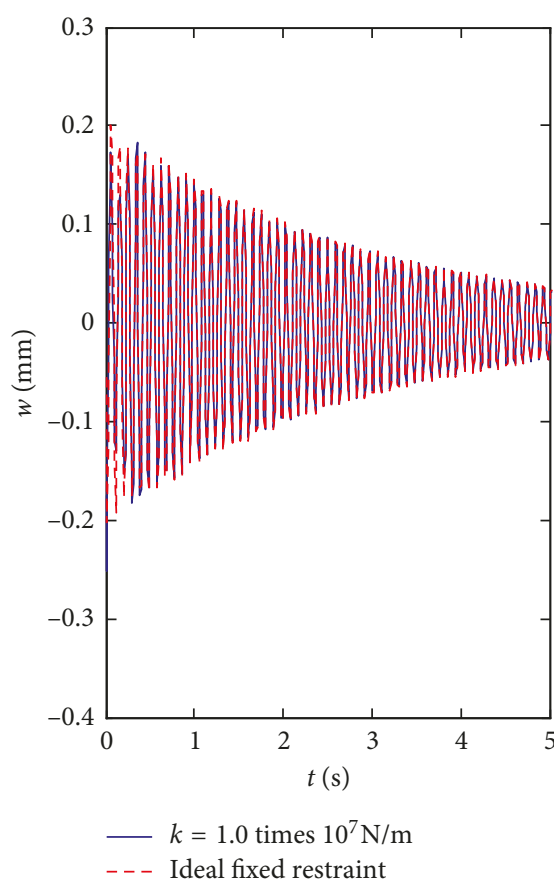

(c)

FIGURE 7: Vibration responses of the FRM with elastic restrained joints.

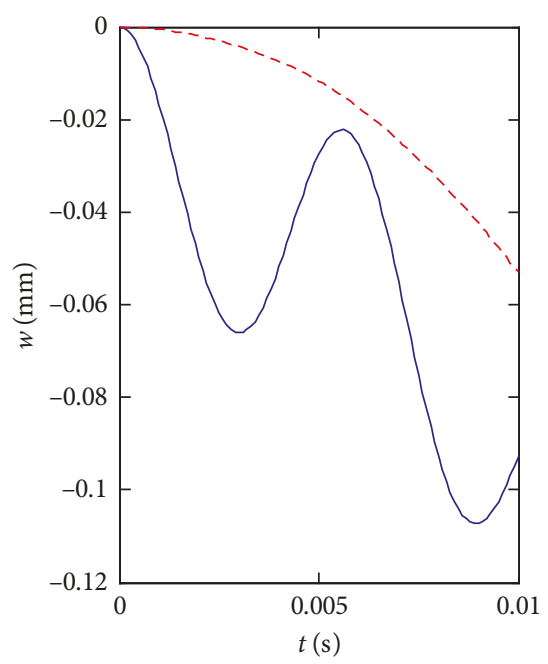

$\quad k=1.0$ times $10^{5} \mathrm{~N} / \mathrm{m}$
- - - Ideal fixed restraint

(a)

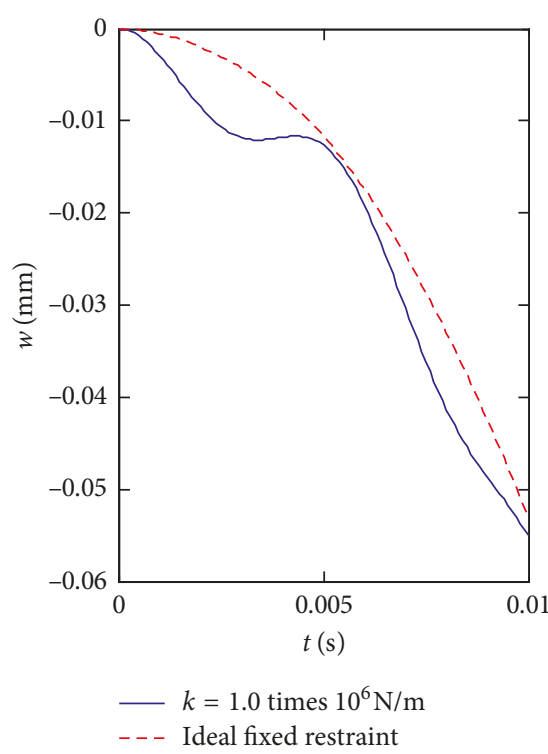

(b)

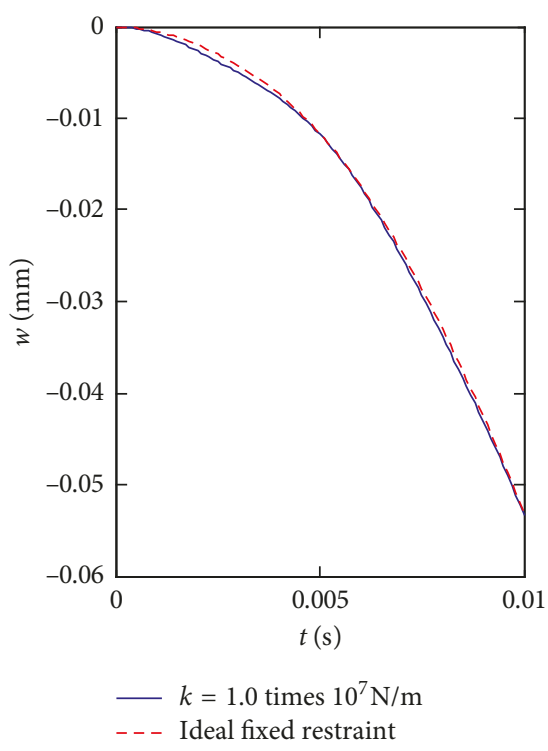

(c)

FIGURE 8: Vibration response displacements of the FRM in the start-up accelerating process.

To further investigate the influence of the ERCJ on the vibration responses, Figure 11 shows the first three orders vibrations of the FRMERCJ and FRMFRCJ. It can be obtained that the third orders vibration displacement of the FRMERCJ is larger than FRMFRCJ while the first- and second-order vibration displacements are similar. This indicates that the elasticity property of the connecting joints significantly influences the higher-order vibration responses, which further reveals that, for higher-order vibrations, the analysis error of the ideal fixed restraint model will be obvious.

\section{Dynamic Prototypal Verification and Restraint Mechanism}

According to Figures 7-10, it can be obtained that the ERCJ have significant influences on the dynamics of the FRM. In 


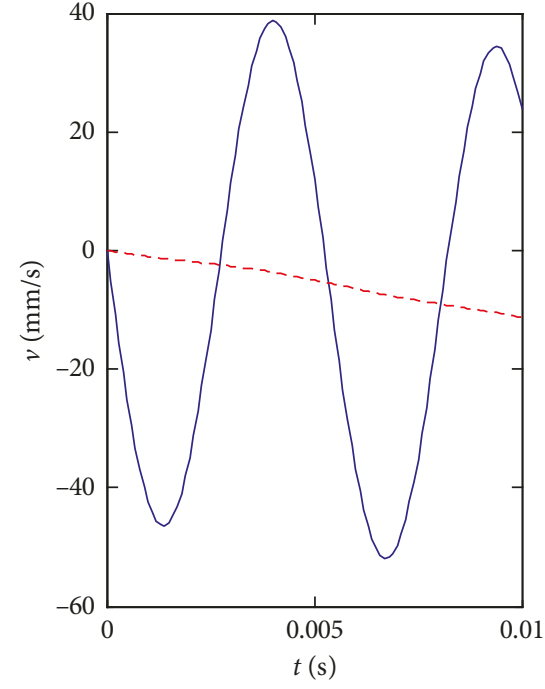

$k=1.0$ times $10^{5} \mathrm{~N} / \mathrm{m}$

- - - Ideal fixed restraint

(a)

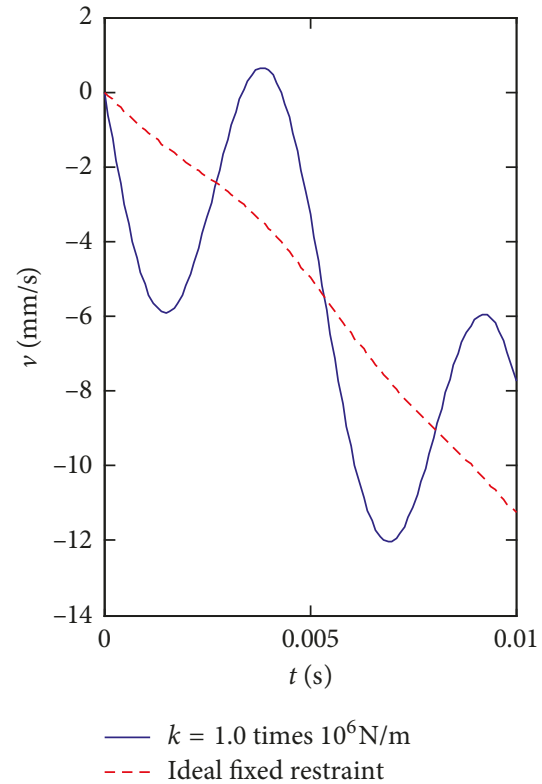

(b)

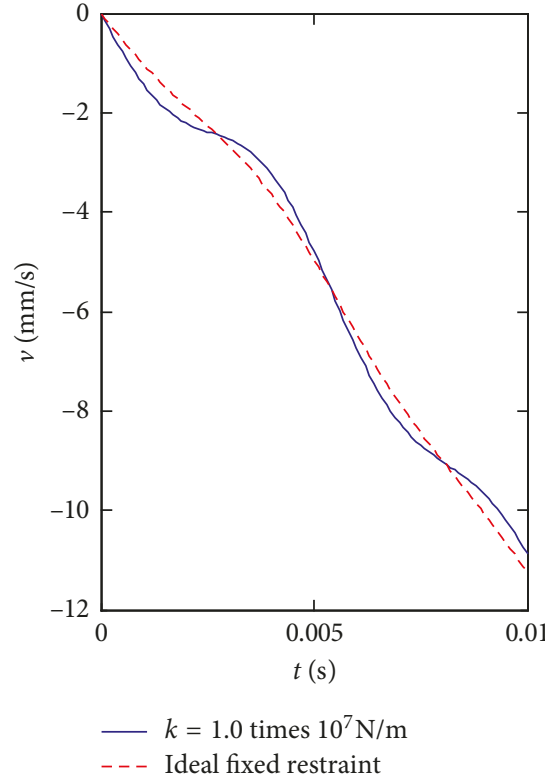

(c)

FIGURE 9: Vibration response velocities of the FRM in the start-up accelerating process.

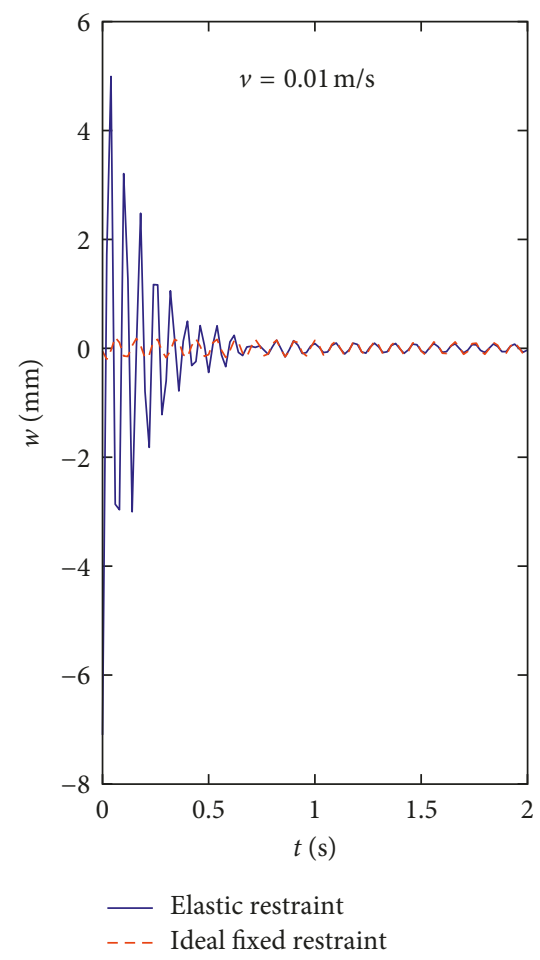

(a)

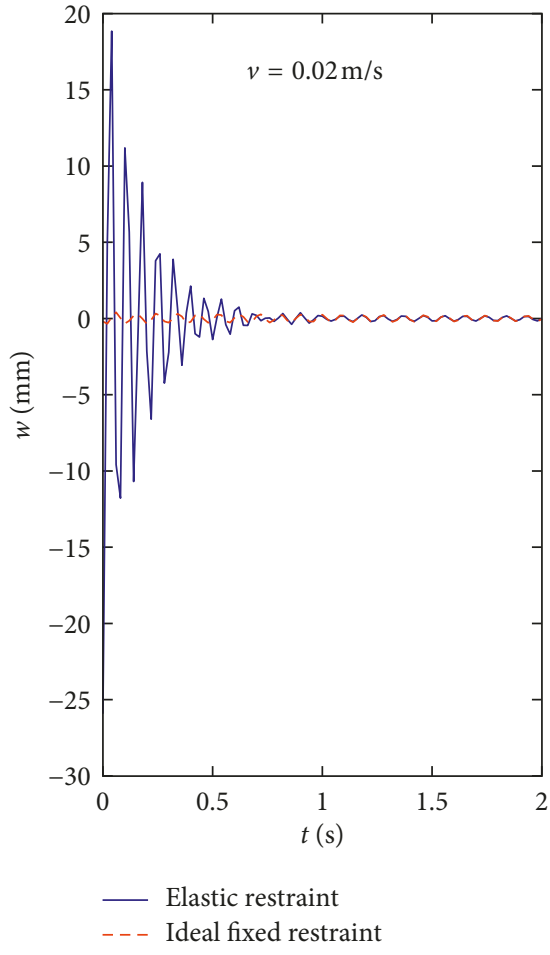

(b)

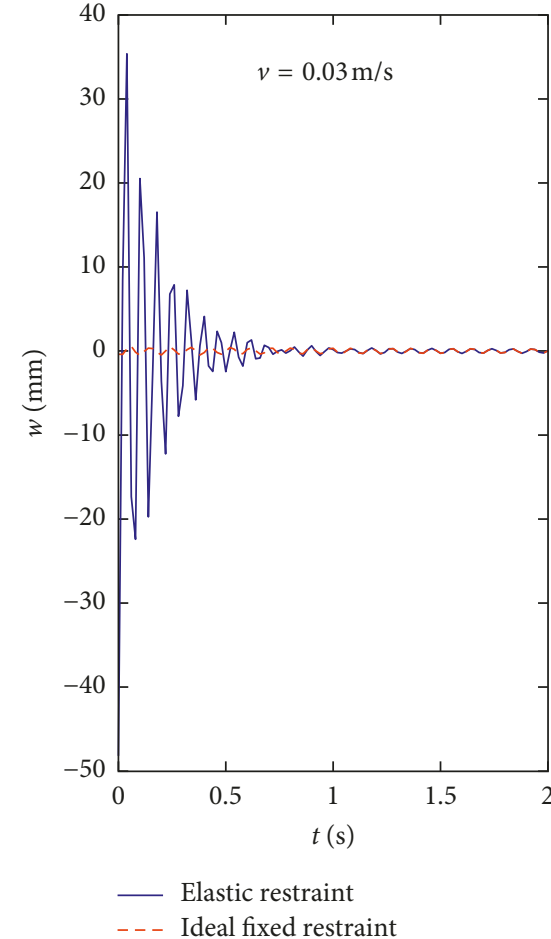

(c)

FIGURE 10: Vibration responses of the FRM with different motion velocities.

particular, the elasticity property has significant influence on the higher-order vibration responses, which are sensitive to motion velocities and will be enhanced under higher velocities especially in the initial stage. In this section, the virtual prototype experiment is conducted to further verify the dynamic model and vibration displacement equation of the FRMERCJ and investigate the restraint mechanism of the ERCJ.

The virtual prototype model is constructed by the multibody dynamics simulation software ADAMS which can create a virtual prototype model of the mechanical 


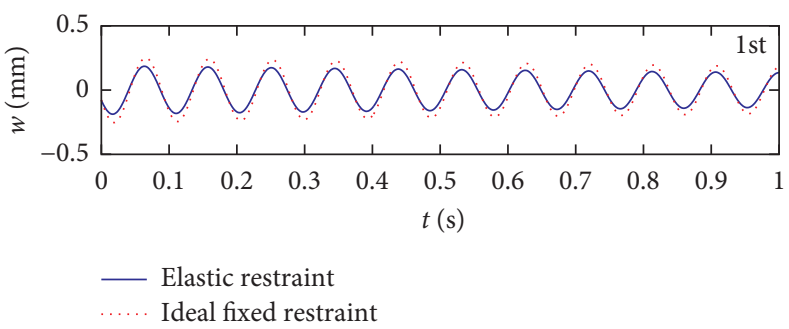

(a)

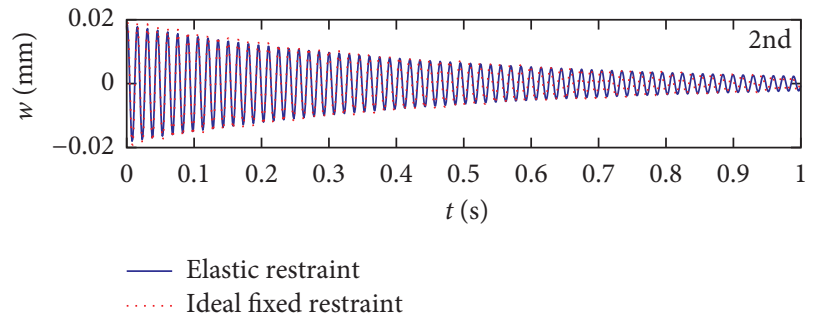

(b)

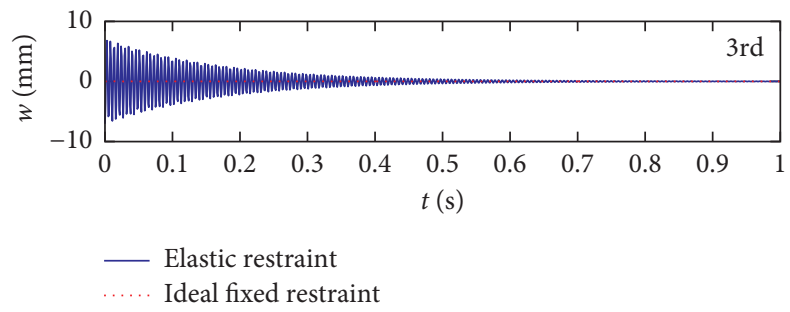

(c)

FIgURE 11: The first three orders vibration responses of the FRM.

system to study the dynamics of the system. The flexible arm is modeled as a flexible body with discrete beam elements while the moving base is modeled as a solid mass. The restrain of the beam element at the end of the flexible body is defined as free, while the restraint of the connecting interface between the flexible body and the solid mass is defined as spring-damping unit with assigned restraint stiffness. Then, movement pair and motion function of the moving base are assigned. The constructed virtual prototype model of the FRMERCJ is shown in Figure 12.

Based on the virtual prototype experiment, the dynamic model and vibration displacement equation of the FRMERCJ are verified firstly. During the verification, the elastic restrained stiffness is assigned as $k=1.0 \times 10^{6} \mathrm{~N} / \mathrm{m}$ and the translating movement velocity is assigned as $v=0.03 \mathrm{~m} / \mathrm{s}$. The vibration responses of the FRMERCJ are shown in Figure 13, which indicates that the numerical results of the dynamic model in this paper are consistent well with the physical model with a same change trend. It should be noted that, due to the nonlinear structural damping parameter of flexible arm and connecting joints, the numerical response amplitude exhibits a difference with the physical model in the initial stage, and with the progress of time, the analysis results are consistent.

To further reveal the dynamic restraint mechanism of the ERCJ, the dynamic response characteristics of the connecting unit are investigated based on the virtual prototype experiments. Figure 14 presents the velocity responses of the connecting unit, it can be seen that the velocity of the connecting unit is not absolutely consistent with the moving base and presents a certain fluctuation in the initial stage; moreover, the lower the restraint stiffness, the more obvious the velocity fluctuation. It is known that if the connecting joints are absolutely fixed restraint, there will be no relative displacements or accelerations between the moving base and the flexible arm. According to Figure 15, however, it reveals that the connecting unit exhibits certain relative acceleration, which is more noticeable for lower restraint stiffness in the initial stage, which indicates that there exists certain relative acceleration between the moving base and the flexible arm. Figure 16 further indicates that the connecting unit conveys certain intrinsic effect force especially for lower restraint stiffness in the initial stage. The conveyed intrinsic effect force will inevitably change the force situation and initial condition of movements, which consequently affects the dynamic characteristics of the flexible arm. These results can illustrate the vibration response characteristics in the initial stage as indicated in Figures 7-10 and reveal the dynamic restraint mechanism of the ERCJ.

\section{Conclusions}

The dynamic characteristics and restraint mechanism of the elastically restrained connecting joints of the FRM are investigated. The elastic restrained region of the connecting joints is defined, and the frequency relationship equation in the elastic restrained region is simultaneously determined and verified. Results indicate that the elasticity property of the connecting joints has significant influence on the dynamic characteristics of the FRM and is sensitive to the motion velocities especially under higher velocities for the higher-order vibrations in the initial stage. The proposed elastic restrained model can accurately describe the elasticity property of the ERCJ. According to the virtual prototype experiment, the dynamic model and vibration equation of the FRMERCJ are verified; moreover, it reveals that the connecting unit conveys certain velocity fluctuation, response displacement, and intrinsic effect force in the initial stage, which inevitably affects the initial conditions of the flexible arm and is more obvious for the lower restraint stiffness. The results in this paper reveal the dynamic characteristics and restraint mechanism of the ERCJ as well as the influence on 


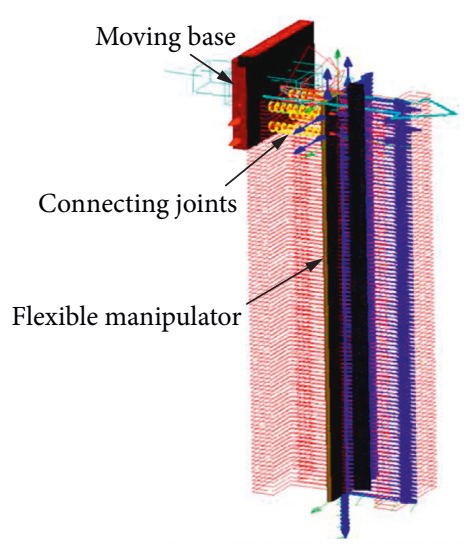

FIGURE 12: Virtual prototype of the FRMERCJ.

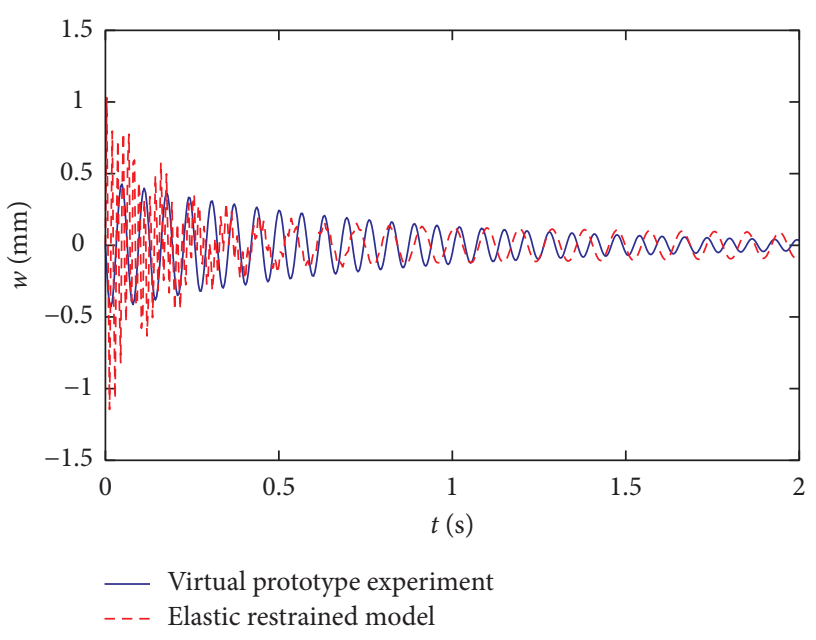

FIgURE 13: Numerical and prototype experimental vibration responses of the FRMERCJ.

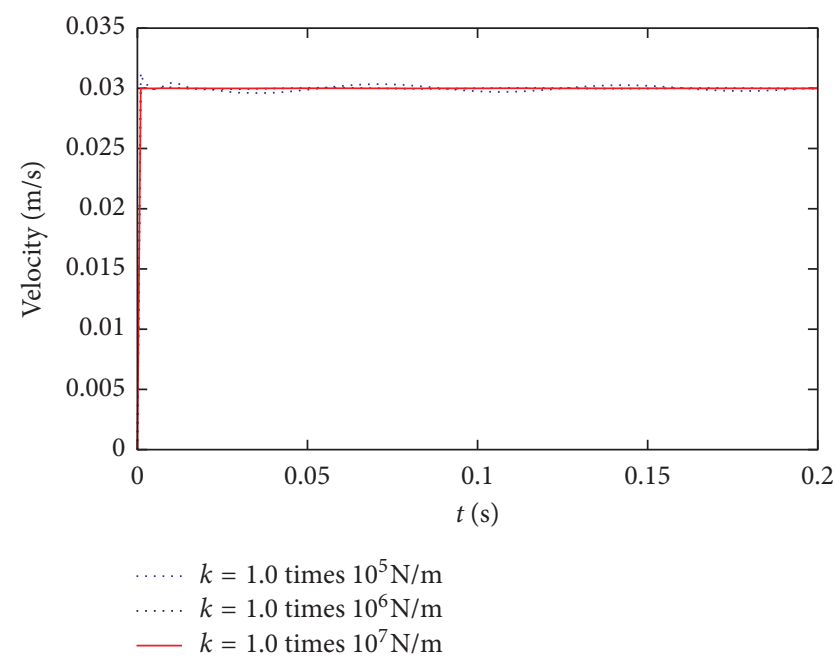

FIGURE 14: Velocity responses of the connecting unit.

the FRM, which should be considered in dynamic analysis and are meaningful for structure design and vibration control of robotic manipulators with bolted connecting joints.

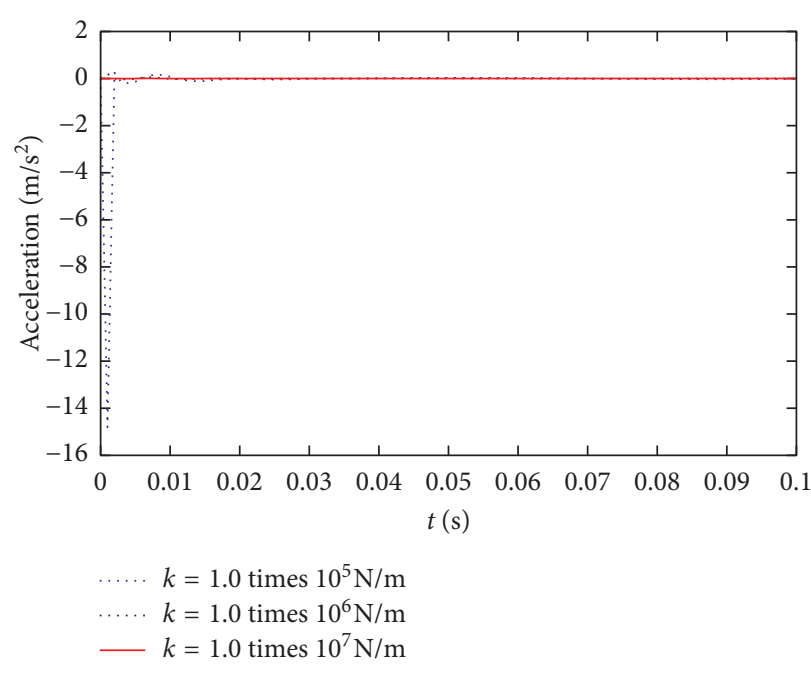

Figure 15: Displacement responses of the connecting unit.

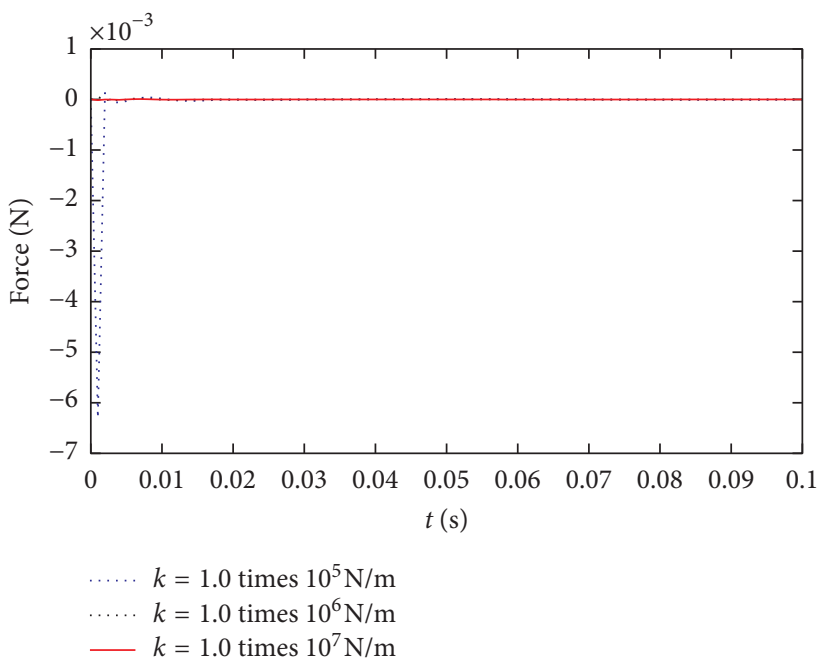

FIGURE 16: Force responses of the connecting unit.

\section{Data Availability}

The data used to support the findings of this study are available from the corresponding author upon request.

\section{Conflicts of Interest}

The authors declare that there are no conflicts of interest regarding the publication of this paper.

\section{Acknowledgments}

This research work is partially supported by the National Natural Science Foundation of China (No. 51805001), Anhui Provincial Natural Science Foundation (No. 1808085QE137), Natural Science Research Project of Higher Education of Anhui Province (No. KJ2017A118), 
and the project funded by China Postdoctoral Science Foundation (No. 2017M612060).

\section{References}

[1] F. Y. Wang and Y. Q. Gao, Advanced Studies of Flexible Robotic Manipulators: Modeling, Design, Control, and Applications, World Scientific, Singapore, 2004.

[2] C. H. Park, D. I. Park, and J. H. Park, "Vibration control of flexible mode for a beam-type substrate transport robot," International Journal of Advanced Robotic Systems, vol. 10, no. 7, pp. 1-7, 2013.

[3] B. Zi, H. Ding, J. Cao, Z. Zhu, and A. Kecskeméthy, "Integrated mechanism design and control forcompletely restrained hybrid-driven based cable parallel manipulators," Journal of Intelligent \& Robotic Systems, vol. 74, no. 3-4, pp. 643-661, 2014.

[4] J. Zhao, S. Yan, and J. Wu, "Analysis of parameter sensitivity of space manipulator with harmonic drive based on the revised response surface method," Acta Astronautica, vol. 98, pp. 86-96, 2014.

[5] S. Seung, P. Liu, S. Park, J. O. Park, and S. Y. Ko, "Single-port robotic manipulator system for brain tumor removal surgery: SiromanS," Mechatronics, vol. 26, pp. 16-28, 2015.

[6] S. E. Butner and M. Ghodoussi, "Transforming a surgical robot for human telesurgery," IEEE Transactions on Robotics and Automation, vol. 19, no. 5, pp. 818-824, 2003.

[7] S. D. Thoppul, J. Finegan, and R. F. Gibson, "Mechanics of mechanically fastened joints in polymer-matrix composite structures: a review," Composites Science and Technology, vol. 69, no. 3-4, pp. 301-329, 2009.

[8] D. Liang and S. F. Yuan, "Decision fusion system for bolted joint monitoring," Shock and Vibration, vol. 2015, Article ID 592043, 11 pages, 2015.

[9] H. N. Rahimi and M. Nazemizadeh, "Dynamic analysis and intelligent control techniques for flexible manipulators: a review," Advanced Robotics, vol. 28, no. 2, pp. 63-76, 2014.

[10] M. Dadfarnia, N. Jalili, Z. Liu, and D. M. Dawson, "An observer-based piezoelectric control of flexible cartesian robot arms: theory and experiment," Control Engineering Practice, vol. 12, no. 8, pp. 1041-1053, 2004.

[11] Z. C. Qiu, "Adaptive nonlinear vibration control of a Cartesian flexible manipulator driven by a ball screw mechanism," Mechanical Systems and Signal Processing, vol. 30, pp. 248266, 2012.

[12] Y. F. Liu, W. Li, X. F. Yang, Y. Q. Wang, M. B. Fan, and G. Ye, "Coupled dynamic model and vibration responses characteristic of a motor-driven flexible manipulator system," $\mathrm{Me}$ chanical Sciences, vol. 6, no. 2, pp. 235-244, 2015.

[13] E. Lu, W. Li, X. F. Yang, M. B. Fan, and Y. F. Liu, "Modelling and composite control of single flexible manipulators with piezoelectric actuators," Shock and Vibration, vol. 2016, Article ID 2689178, 14 pages, 2016.

[14] Y. Chen, Q. Gao, and Z. Q. Guan, "Self-loosening failure analysis of bolt joints under vibration considering the tightening process," Shock and Vibration, vol. 2017, Article ID 2038421, 15 pages, 2017.

[15] W. Chen, "Dynamic modeling of multi-link flexible robotic manipulators," Computers \& Structures, vol. 79, no. 2, pp. 183-195, 2001.

[16] Y. Liu, W. Li, Y. Wang, X. Yang, and J. Ju, "Dynamic model and vibration power flow of a rigid-flexible coupling and harmonicdisturbance exciting system for flexible robotic manipulator with elastic joints," Shock and Vibration, vol. 2015, Article ID 541057, 10 pages, 2015.

[17] B. Subudhi and A. S. Morris, "Dynamic modelling, simulation and control of a manipulator with flexible links and joints," Robotics and Autonomous Systems, vol. 41, no. 4, pp. 257-270, 2002.

[18] Z. J. Qian and D. G. Zhang, "Impact dynamics of multi-link robots with link and joint flexibility," Applied Mechanics and Materials, vol. 226-228, pp. 685-692, 2012.

[19] L. Malgaca, H. Dogan, M. Akdağ, Ş. Yavuz, M. Uyar, and B. Bidikli, "Effect of joint flexibility on vibration characteristics of a composite box manipulator," Composite Structures, vol. 183, pp. 271-277, 2018.

[20] J. Wei, D. Cao, L. Liu, and W. Huang, "Global mode method for dynamic modeling of a flexible-link flexible-joint manipulator with tip mass," Applied Mathematical Modelling, vol. 48 , pp. 787-805, 2017.

[21] J. T. Agee, Z. Bingul, and S. Kizir, "Trajectory and vibration control of a single-link flexible-joint manipulator using a distributed higher-order differential feedback controller," Journal of Dynamic Systems, Measurement, and Control, vol. 139, no. 8, article 081006, 2017.

[22] T. N. Guo, L. Li, L. G. Cai, and Y. S. Zhao, "Alternative method for identification of the dynamic properties of bolted joints," Journal of Mechanical Science and Technology, vol. 26, no. 10, pp. 3017-3027, 2012.

[23] J. H. Wang and S. C. Chuang, "Reducing errors in the identification of structural joint parameters using error functions," Journal of Sound and Vibration, vol. 273, no. 1-2, pp. 295-316, 2004.

[24] Y. F. Liu, W. Li, X. F. Yang, and M. B. Fan, "Mode and vibration characteristics of a flexible manipulator with elastic restraint joint," Journal of Vibroengineering, vol. 16, no. 2, pp. 933-944, 2014.

[25] S. R. Singiresu, Mechanical Vibrations, Pearson Education Inc., London, UK, 5th edition, 2010.

[26] X. Mei, Analytical Mechanics, Beijing Institute of Technology Press, Beijing, China, 2013.

[27] S. O. R. Moheimani and A. J. Fleming, Fundamentals of Pezoelectricity. Piezoelectric Transducers for Vibration Control and Damping, Springer Science \& Business Media, London, UK, 2006. 


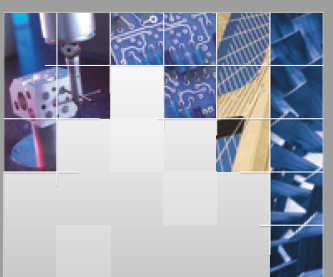

\section{Enfincering}
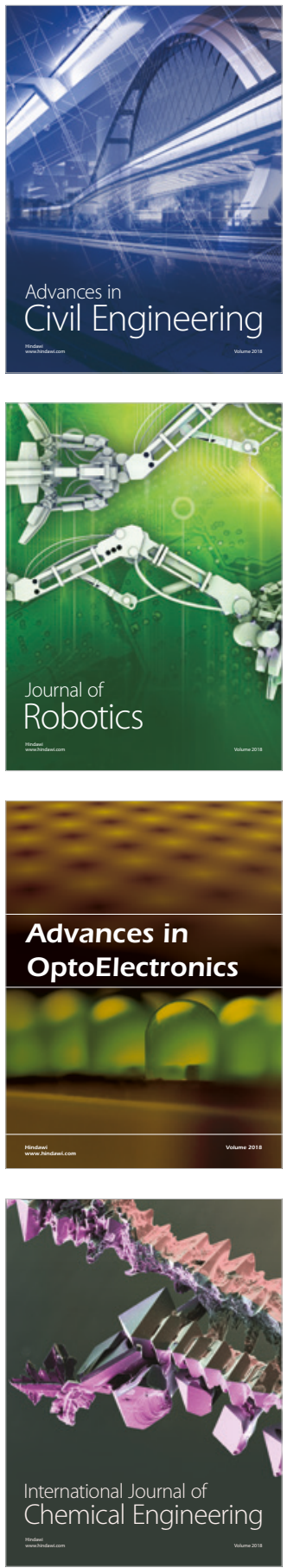

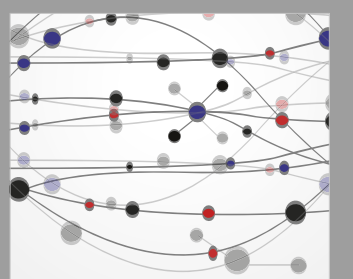

\section{Rotating \\ Machinery}

The Scientific World Journal

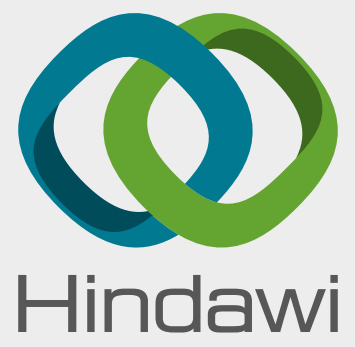

Submit your manuscripts at

www.hindawi.com
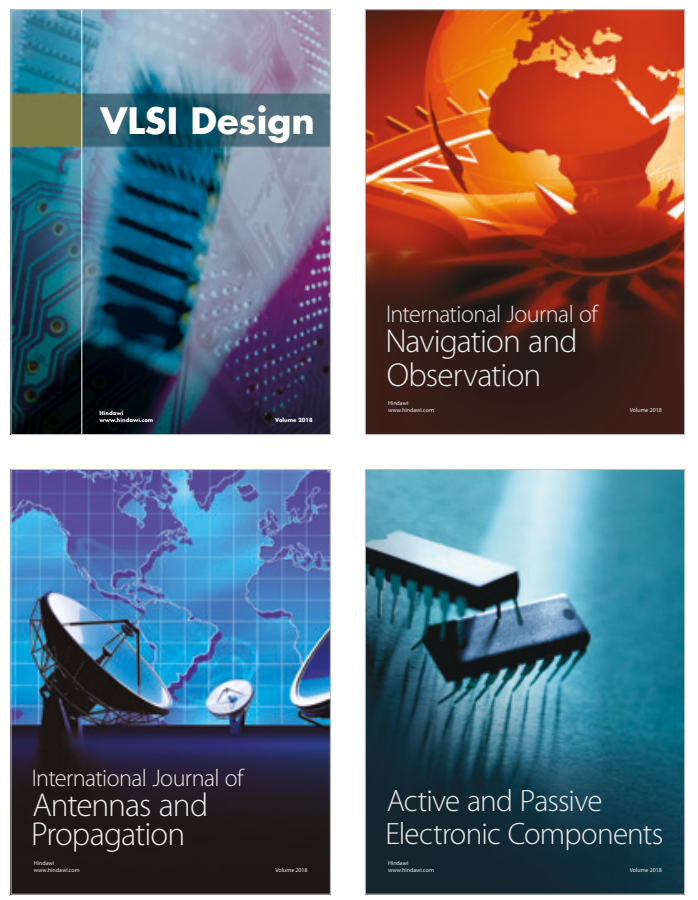
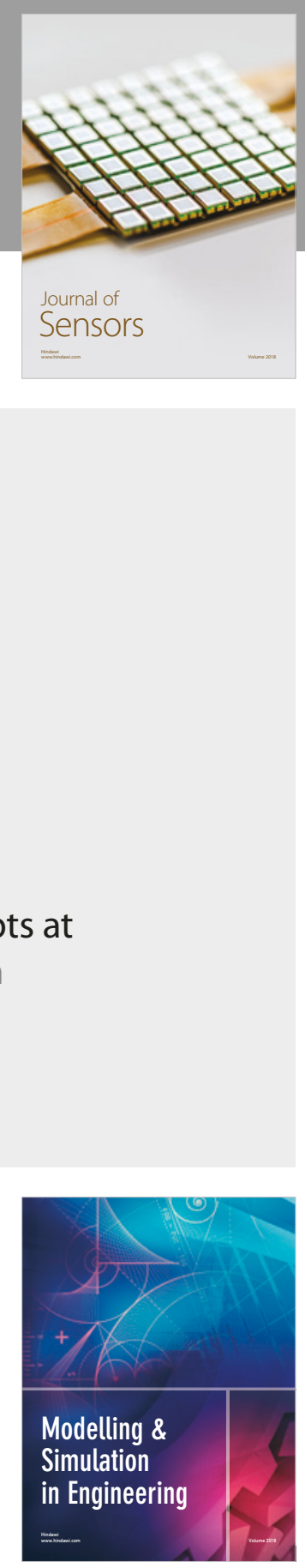

\section{Advances \\ Multimedia}
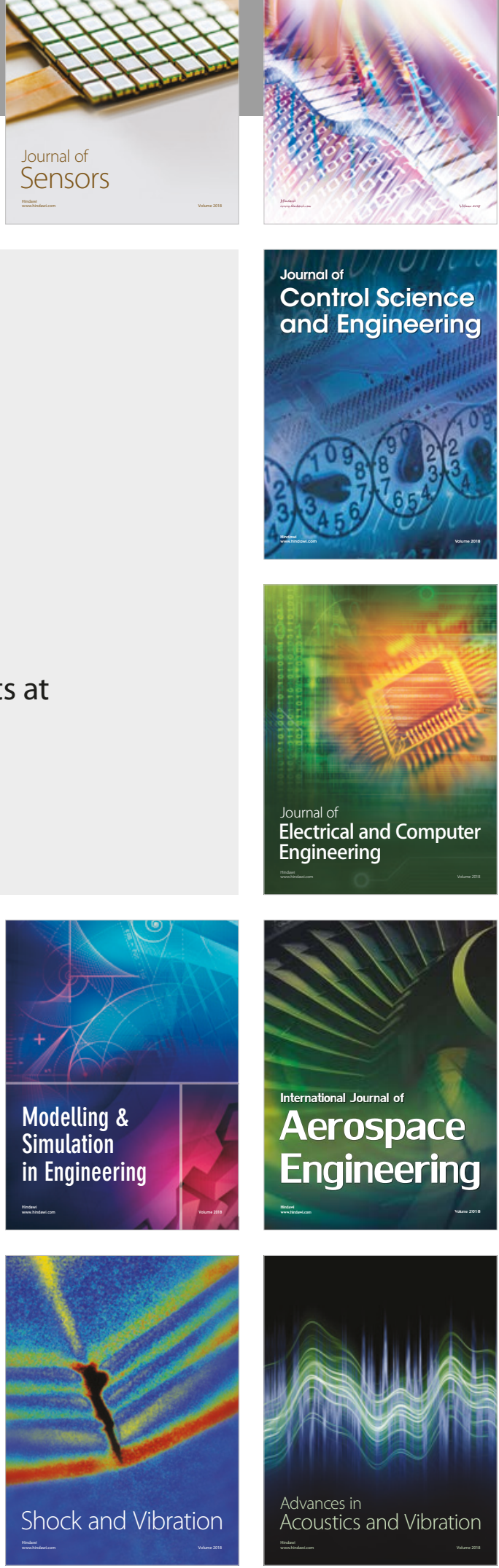